\title{
Robotic system for inspection of test objects with unknown geometry using NDT methods
}

Alina-A Brenner, Tariq. P. Sattar

Department of Electrical, Computer and Communications Engineering, London South Bank University, 103 Borough Road, London SE1 0AA email: brennea@1sbu.ac.uk, sattartp@1sbu.ac.uk

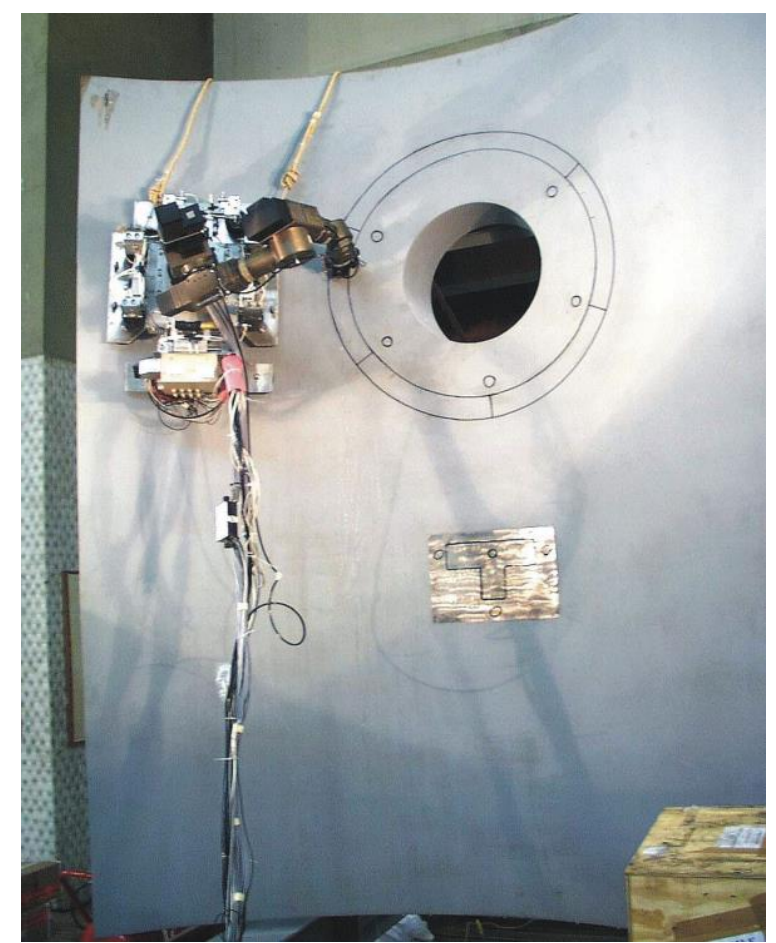

Figure 1: The portable non-destructive testing robot carried by a wall climbing robot on a pressure vessel mock up to inspect nozzle welds

The aim of this work is to develop a portable Non-Destructive Testing (NDT) robotic system that can be carried by CLAWAR to evaluate defects in geometrically complex surfaces and industrial products.

This paper builds on a previous work presented during CLAWAR 2006. It presents further progress and discusses new results on the subject of surface adaptation and NDT ultrasonic data acquisition. The focus is on the behaviour of the system and the quality of data gathered by the NDT inspection system when affected by the robot's motion trajectory. 


\section{Application of wall climbing robots}

Many industrial NDT applications, such as on aircraft structures, nuclear pressure vessels, off-shore oil platforms, large bridges, hulls of ships or chemical storage tanks, etc., require climbing and walking robots so that access to structures in hazardous environments can be provided. The automated maintenance and inspection, using robots, is indispensable to the safety of the workers. In addition, automation promises to improve the quality of defect data and hence detection by utilising the greater repeatability, accuracy and rigidity of robots.

Also, the traditional ways of manual inspection are also very costly and can be made more cost-effective if automated.

Wall-climbing robots are increasingly employed to provide access to large vertical structures to evaluate the condition of the structure using Non-Destructive Technology. The wall-climbing robot, in combination with a scanning robot arm, can operate on structures with more complex geometry and provide accuracy; thereby creating a flexible all-round inspection system which can adapt to diverse environments.

The seven axis arm shown in figure 1 has been especially developed to be carried on a mobile robot. It is designed to form a lightweight system which at the same time features the dexterity and flexibility approaching that of a human arm. Its control system offers the ability to adaptively scan complex surfaces that are not precisely known via force sensor feedback.

The resulting robotic system contributes to the progress of automated NDT as it provides the possibility of remote inspection of complex pieces in various hazardous environments.

Results of robotic trajectory planning and robotic motion control of this system are reported in $[1,2]$ that investigate automated ultrasonic testing by incorporating in real time the position data with the NDT data.

In this paper, the difference in quality of defect data during manual inspection and automated inspection will be compared. Major focus points are the quality of NDT data, the hit rate of defect detection, the occurrence of false calls as well as improvement in surface contact endurance.

Tests have been performed on complex shaped turbine blades using eddy currents. The challenge is to be able to follow the surface by keeping the NDT probe normal to the surface while maintaining a constant contact force with it. One approach to maintaining a constant contact force and angle to the object was to use a secure contact using permanent magnets and a force adapting control of the manipulator which resulted in improvements in the quality of inspection compared to manual inspection.

Furthermore, a closer look at purely force control adaptation using a rigid probe holder has been investigated. For this purpose, a program handling different parallel processes has been designed to investigate the relation between adaptation forces and 
NDT data. A force sensor from JR3 is mounted on the robot's wrist. This will provide surface adaptation in real time thus allowing task-based control strategies to be employed and will contribute towards the assurance of continuous NDT data recording during motion on complex and unknown surfaces. Results of these tests will be presented.

[1] Alina-A Brenner, T. P. Sattar (2006), Portable 7 DOF Scanning Arm For Nondestructive Inspection Of Objects With Unknown Geometry, Proceedings of the 9th International Conference on Climbing and Walking Robots and the Support Technologies for Mobile Machines, September 12 - 14, 2006, Brussels, Belgium, pp 527-532.

[2] Sattar T.P and Brenner A. (2005) Development Of A Seven-Axis Scanning Arm For Remote Non-Destructive Testing That Can Be Carried By Climbing And Walking Robots, Proceedings of the $8^{\text {th }}$ International Conference on Climbing and Walking Robots and the Support Technologies for Mobile Machines (CLAWAR 2005), Editors Tokhi, Virk and Hossain, ISBN-10 3-540-26413-2, Springer, ISBN-13 978-3-540-26413-2, pp 943-950

\section{Reference Information for Submission \#61}

Title: Robotic system for inspection of test objects with unknown geometry using NDT methods

Authors: Alina Alexandra Brenner and Tariq Pervez Sattar

Passcode: 61X-J9G4J9B4C7

Confirmation 61

Number: 6 